\title{
TWISTED BURNSIDE THEOREM FOR TYPE II 1 GROUPS: AN EXAMPLE
}

\author{
Alexander Fel'shtyn, Evgeniu Troitsky, and Anatoly Vershik \\ Abstract. The purpose of the present paper is to discuss the following conjecture of \\ Fel'shtyn and Hill, which is a generalization of the classical Burnside theorem: \\ Let $G$ be a countable discrete group, $\phi$ its automorphism, $R(\phi)$ the number of $\phi$ - \\ conjugacy classes (Reidemeister number), $S(\phi)=\# \operatorname{Fix}(\widehat{\phi})$ the number of $\phi$-invariant \\ equivalence classes of irreducible unitary representations. If one of $R(\phi)$ and $S(\phi)$ is \\ finite, then it is equal to the other. \\ This conjecture plays a very important role in the theory of twisted conjugacy classes \\ having a long history (see [14], [4]) and has very serious consequences in Dynamics, \\ while its proof needs rather fine results from Functional and Non-commutative Harmonic \\ Analysis. It was proved for finitely generated groups of type I in [10]. \\ In the present paper this conjecture is disproved for non-type I groups. More precisely, \\ an example of a group and its automorphism is constructed such that the number of fixed \\ irreducible representations is greater than the Reidemeister number. But the number of \\ fixed finite-dimensional representations (i.e. the number of invariant finite-dimensional \\ characters) in this example coincides with the Reidemeister number. \\ The directions for search of an appropriate formulation are indicated (another defi- \\ nition of the dual object).
}

\section{Introduction: motivation, history, and current state}

Definition 1.1. Let $G$ be a countable discrete group and $\phi: G \rightarrow G$ an endomorphism. Two elements $x, x^{\prime} \in G$ are said to be $\phi$-conjugate or twisted conjugate, iff there exists $g \in G$ with

$$
x^{\prime}=\operatorname{gx\phi }\left(g^{-1}\right) .
$$

We shall write $\{x\}_{\phi}$ for the $\phi$-conjugacy or twisted conjugacy class of the element $x \in G$. The number of $\phi$-conjugacy classes is called the Reidemeister number of an endomorphism $\phi$ and is denoted by $R(\phi)$. If $\phi$ is the identity map then the $\phi$-conjugacy classes are the usual conjugacy classes in the group $G$.

If $G$ is a finite group, then the classical Burnside theorem (see e.g. [15, p. 140]) says that the number of classes of irreducible representations is equal to the number of conjugacy classes of elements of $G$. Let $\widehat{G}$ be the unitary dual of $G$, i.e. the set of equivalence classes of unitary irreducible representations of $G$.

Received by the editors April 26, 2005.

This a joint research completed during the stay at Max-Planck-Institut für Mathematik (Bonn) in Spring 2004 in partial relation to the activity on Algebraic and Topological Dynamics. The authors are grateful to MPIM for support and hospitality.

The second author is partially supported by RFBR Grant 05-01-00293 and Grant РНП.2.1.1.5055.

The third author is partially supported by RFBR Grant 05-01-00899 (Russia) and CRDF Grant RUM-2622.ST.04 (US).

The authors are indebted to the referee for very useful suggestions. 
Therefore, by the Burnside's theorem, if $\phi$ is the identity automorphism of any finite group $G$, then we have $R(\phi)=\# \operatorname{Fix}(\widehat{\phi})$, where $\widehat{\phi}[\rho]=[\rho \circ \phi]$.

One of the main achievements in the field till now is the following result.

Theorem 1.2 ([10]). Let $G$ be a finitely generated discrete group of type I, $\phi$ one of its endomorphism, $R(\phi)$ the number of $\phi$-conjugacy classes, and $S(\phi)=\# \operatorname{Fix}(\widehat{\phi})$ the number of $\widehat{\phi}$-invariant equivalence classes of irreducible unitary representations. If one of $R(\phi)$ and $S(\phi)$ is finite, then it is equal to the other.

The research is motivated not only by a natural desire to extend the classical Burnside theorem to the case of infinite groups and twisted conjugacy classes, but also by dynamical applications. Namely, the identification of Reidemeister number with a number of fixed points in a natural way, has some very interesting consequences.

More precisely, let $\mu(d), d \in \mathbb{N}$, be the Möbius function, i.e.

$$
\mu(d)= \begin{cases}1 & \text { if } d=1, \\ (-1)^{k} & \text { if } d \text { is a product of } k \text { distinct primes } \\ 0 & \text { if } d \text { is not square }- \text { free }\end{cases}
$$

Theorem 1.3 (Congruences for the Reidemeister numbers [10]). Let $\phi: G \rightarrow G$ be an endomorphism of a countable discrete group $G$ such that all numbers $R\left(\phi^{n}\right)$ are finite and let $H$ be a subgroup of $G$ with the properties

$$
\begin{gathered}
\phi(H) \subset H, \\
\forall x \in G \exists n \in \mathbb{N} \text { such that } \phi^{n}(x) \in H .
\end{gathered}
$$

If for the pair $\left(H, \phi^{n}\right)$ the twisted Burnside theorem holds, i.e. this pair satisfies the conclusion of Theorem 1.2 for any $n \in \mathbb{N}$, then one has for all $n$,

$$
\sum_{d \mid n} \mu(d) \cdot R\left(\phi^{n / d}\right) \equiv 0 \bmod n
$$

These theorems were proved previously in a special case of a direct sum of an Abelian finitely generated group and finite group [6, 7]. Remember that the difference between result of $[6,7]$ and Theorem 1.2 is not so big because by theorem of E.Thoma a countable finitely generated group $G$ is a group of type $\mathrm{I}$ iff it is a finite extension of an Abelian group. The conjecture under discussion was formulated in [6]. We refer to $[4,10]$ for more detail and topological applications.

On the other hand, one can introduce the number $R_{*}(\phi)$ of "Reidemeister classes related to twisted invariant functions on $G$ from the Fourier-Stieltjes algebra $B(G)$ ", or more precisely, the dimension of the space of twisted invariant functions on $G$ which can be extended up to bounded functionals on the group algebra $C^{*}(G)$. Let $S_{*}(\phi)$ be the sum of codimensions of subspaces $L_{I} \subset C^{*}(G) / I$, where $L_{I}$ is generated by elements of the form $a-L_{g} a L_{\phi\left(g^{-1}\right)}$ and $I$ runs over the Glimm spectrum $T$ of $G$. Let us remind this notion. Let $Z$ be the center of a $\mathrm{C}^{*}$-algebra $A$ and $\widehat{Z}$ its space of maximal ideals equipped with the standard topology. If $I \in \mathcal{P}:=\operatorname{Prim}(A)$ (the space of kernels of unitary irreducible representations), then $Z \cap I \in \widehat{Z}$ (this follows from the fact that the restriction onto $Z$ of an irreducible representation with kernel $I$ gives rise to a homomorphism $Z \rightarrow \mathbb{C}$, and hence is a maximal ideal $Z \cap I)$. We obtain a map $f: \mathcal{P} \rightarrow \widehat{Z}$. Suppose $T:=f(\mathcal{P})$. For each $x \in T$ consider the ideal $I_{x}:=\cap I, f(I)=x$, 
(Glimm ideal) and the field of algebras $A / I_{x}$. We have the map $a \mapsto\left\{x \mapsto a+I_{x}\right\}$ from the algebra $A$ to the algebra of sections of the mentioned field. An important result of [3] asserts that this map is an isomorphism. The map $f: \mathcal{P} \rightarrow T$ is universal with respect to continuous maps $g: \mathcal{P} \rightarrow S$ to Hausdorff spaces, i.e. any such map can be represented under the form $h \circ f$ for some continuous $h: T \rightarrow S$. The space $T$ is compact for a unital algebra.

We call $S_{*}(\phi)$ the number of generalized fixed points of $\widehat{\phi}$ on the Glimm spectrum of $G$.

Theorem 1.4 (weak twisted Burnside theorem, [19]). The number $R_{*}(\phi)$ is equal to the number $S_{*}(\phi)$ of generalized fixed points of $\widehat{\phi}$ on the Glimm spectrum of $G$, if one of $R_{*}(\phi)$ and $S_{*}(\phi)$ is finite.

This result allows to obtain the strong form of twisted Burnside theorem $R(\phi)=$ $S(\phi)$ in a number of cases.

The interest in twisted conjugacy relations has its origins, in particular, in the Nielsen-Reidemeister fixed point theory (see, e.g. [14, 4]), in Selberg theory (see, eg. $[18,1]$ ), and Algebraic Geometry (see, e.g. [13]).

The congruences give some necessary conditions for the realization problem for Reidemeister numbers in topological dynamics.

Let us remark that it is known that the Reidemeister number of an endomorphism of a finitely generated Abelian group is finite iff 1 is not in the spectrum of the restriction of this endomorphism to the free part of the group (see, e.g. [14]). The Reidemeister number is infinite for any automorphism of a non-elementary Gromov hyperbolic group $[11,16]$ as well as for any injective endomorphism of BaumslagSolitar group [5] (see also [8]).

The main result of the present paper is the following statement.

Theorem 1.5. There exists an amenable solvable group $G$ not of type $I$ and its automorphism $\phi$ such that its Reidemeister number $R(\phi)$ is finite but does not coincide with the number $S(\phi)$ of fixed points of $\widehat{\phi}$ on $\widehat{G}$.

This example is very important for the further attack onto the problem, because this group is "situated between" the groups of type I, for which the conjecture is true [10], and Gromov hyperbolic groups, for which Reidemeister numbers are always infinite.

The ideas for the further study arising from the example from this paper and from [19] are discussed in the last section below.

\section{Some technical preliminaries}

Let $G$ be a semidirect product of $\mathbb{Z}^{2}$ and $\mathbb{Z}$ by Anosov automorphism $\alpha$ with the matrix $A=\left(\begin{array}{ll}2 & 1 \\ 1 & 1\end{array}\right)$ (of course, our results remain valid for any hyperbolic element of $S L(2, \mathbb{Z})$ to use as $A)$. It consists by the definition of triples $((m, k), n)$ of integers with the following multiplication low:

$$
((m, k), n) *\left(\left(m^{\prime}, k^{\prime}\right), n^{\prime}\right)=\left((m, k)+\alpha^{n}\left(m^{\prime}, k^{\prime}\right), n+n^{\prime}\right) .
$$


In particular,

$$
((m, k), 0) *((0,0), n)=((m, k), n) .
$$

Elements of $G$ can be written also as matrices

$$
\left[\begin{array}{cc}
\alpha^{n} & x \\
0 & 1
\end{array}\right], \quad n \in \mathbb{Z}, \quad x=(m, k) \in \mathbb{Z}^{2} .
$$

The inverse of $((m, k), n)$ is $\left(-\alpha^{-n}(m, k),-n\right)$. Indeed,

$$
((m, k), n) *\left(-\alpha^{-n}(m, k),-n\right)=\left((m, k)-\alpha^{n} \alpha^{-n}(m, k), n-n\right)=((0,0), 0) .
$$

The group $G$ is a solvable (hence, amenable) group which is not of type I. Its regular representation is factorial.

Let us define an automorphism $\phi: G \rightarrow G$ by

$$
\phi((m, k), n)=((k,-m),-n),
$$

i.e. the action on $\mathbb{Z}^{2}$ is defined by automorphism $\mu$ with the matrix $M=\left(\begin{array}{cc}0 & 1 \\ -1 & 0\end{array}\right)$, and on $\mathbb{Z}$ by $n \mapsto-n$. The map $\phi$ is clearly a bijection,

$$
\begin{aligned}
& \phi\left(((m, k), n) *\left(\left(m^{\prime}, k^{\prime}\right), n^{\prime}\right)=\phi\left((m, k)+\alpha^{n}\left(m^{\prime}, k^{\prime}\right), n+n^{\prime}\right)\right) \\
& =\left((k,-m)+\mu \alpha^{n}\left(m^{\prime}, k^{\prime}\right),-n-n^{\prime}\right), \\
& \begin{aligned}
& \phi((m, k), n) * \phi\left(\left(\left(m^{\prime}, k^{\prime}\right), n^{\prime}\right)=((k,-m),-n) *\left(\left(k^{\prime},-m^{\prime}\right),-n^{\prime}\right)\right. \\
&=\left((k,-m)+\alpha^{-n}\left(k^{\prime},-m^{\prime}\right),-n-n^{\prime}\right) .
\end{aligned}
\end{aligned}
$$

Hence, to prove that $\phi$ is an automorphism, we need $\mu \alpha^{n}=\alpha^{-n} \mu$. This follows from $\mu \alpha=\alpha^{-1} \mu$. The further results in this direction can be found in [12].

One can hope to find $\widehat{\phi}$-fixed irreducible representations (at least finite-dimensional ones) from those orbits of $\alpha$ on the dual torus $\mathbb{T}^{2}$ to the normal subgroup $\mathbb{Z}^{2}$, which are invariant under the dual action of $\mu$, using appropriate cocycles. This will be done in the last section. We will find four such representations and we will explain why that is all. Some motivation for this way of construction can be found in $[2, \mathrm{Ch}$. 17 , $\S 1]$.

\section{Description of Reidemeister classes}

Let us find the Reidemeister classes of $\phi$, i.e. the classes of the equivalence relation $h \sim g h \phi\left(g^{-1}\right)$. We will prove the following statement.

Lemma 3.1. For the above $G$ and $\phi$ one has $R(\phi)=4$.

Also we will obtain a description of these four Reidemeister classes.

For $h=((m, k), n)$ and $g=((x, y), z)$ the right hand side of the relation takes the following form:

$$
\begin{aligned}
& \left((x, y)+\alpha^{z}(m, k), z+n\right) *\left(-\mu \alpha^{-z}(x, y), z\right)= \\
& \left((x, y)+\alpha^{z}(m, k)-\alpha^{z+n} \mu \alpha^{-z}(x, y), 2 z+n\right)= \\
& =\left(\alpha^{z}\left\{(m, k)+\left(\operatorname{Id}-\alpha^{n} \mu\right) \alpha^{-z}(x, y)\right\}, 2 z+n\right) .
\end{aligned}
$$

Let us call level $n$ (of $G$ ) the coset $L_{n}$ of $\mathbb{Z}^{2} \subset G$ of all elements of the form $((m, k), n$ ). Let us first take an element $((m, k), 0)$ from the level 0 and describe elements from 
the same level, being equivalent to it. By (1) in this case $z=n=0$ and they have the form

$$
((m, k)+(\operatorname{Id}-\mu)(x, y), 0)=((m+(x-y), k+(x+y)), 0),
$$

where Id $-\mu$ has the matrix $\left(\begin{array}{cc}1 & -1 \\ 1 & 1\end{array}\right)$. Hence, the level 0 has intersections with 2 Reidemeister classes, say, $B_{1}$ and $B_{2}$. The first intersection $B_{1} \cap L_{0}$ is formed by elements $((u, v), 0)$ with odd $u+v$, and $B_{2} \cap L_{0}$ - with even $u+v$. The elements from the other levels, which are equivalent to $((m, k), 0)$, have the form

$$
\left(\alpha^{z}\left\{(m, k)+(\operatorname{Id}-\mu) \alpha^{-z}(x, y)\right\}, 2 z\right) .
$$

This means that $B_{1}$ and $B_{2}$ enter only even levels. Also, since $\alpha$ is an automorphism, we can rewrite (2) as

$$
\left(\alpha^{z}\{(m, k)+(\operatorname{Id}-\mu)(u, v)\}, 2 z\right) .
$$

with arbitrary integers $u$ and $v$. This means, that the intersections $B_{i} \cap L_{2 z}$ have the form $\alpha^{z}\left(B_{i}\right), i=1,2$. In particular, the other Reidemeister classes do not enter even levels.

In a similar way, the elements of $L_{1}$ equivalent to $((m, k), 1)$ have the form

$$
((m, k)+(\operatorname{Id}-\alpha \mu)(x, y), 1)=((m+(2 x-2 y), k+x), 1) .
$$

This means, that $L_{1}$ enters 2 classes: $B_{3}$ is formed by elements with even first coordinate, and $B_{4}$ - with odd one. The elements from the other levels, which are equivalent to $((m, k), 1)$, have the form

$$
\left(\alpha^{z}\left\{(m, k)+(\operatorname{Id}-\alpha \mu) \alpha^{-z}(x, y)\right\}, 2 z+1\right) .
$$

Since $\alpha$ is an automorphism, we can rewrite (4) as

$$
\left(\alpha^{z}\{(m, k)+(\operatorname{Id}-\alpha \mu)(u, v)\}, 2 z+1\right) .
$$

with arbitrary integers $u$ and $v$. This means, that the intersections $B_{i} \cap L_{2 z+1}$ have the form $\alpha^{z}\left(B_{i}\right), i=3,4$. In particular, these four classes cover $G$ and $R(\phi)=4$.

To obtain a complete description of $B_{i}$ let us remark that directly from the definition of $\alpha$

$$
\alpha(x, y)=(2 x+y, x+y)
$$

one has the following properties:

- $\alpha$ maps the set of elements with an even (resp., odd) sum of coordinates onto the set of elements with an even (resp., odd) second coordinate,

- $\alpha$ maps the set of elements with an even (resp., odd) second coordinate onto the set of elements with an even (resp., odd) first coordinate,

- $\alpha$ maps the set of elements with an even (resp., odd) first coordinate onto the set of elements with an even (resp., odd) sum of coordinates.

Hence, the elements $((m, k), n)$ in intersections $B_{i} \cap L_{j}$ are of the form 


\begin{tabular}{|c|c|c|c|c|}
\hline$i$ & 1 & 2 & 3 & 4 \\
\hline \hline$j \equiv 0 \bmod 6$ & $m+k$ is even & $m+k$ is odd & $\emptyset$ & $\emptyset$ \\
\hline$j \equiv 1 \bmod 6$ & $\emptyset$ & $\emptyset$ & $m$ is even & $m$ is odd \\
\hline$j \equiv 2 \bmod 6$ & $k$ is even & $k$ is odd & $\emptyset$ & $\emptyset$ \\
\hline$j \equiv 3 \bmod 6$ & $\emptyset$ & $\emptyset$ & $m+k$ is even & $m+k$ is odd \\
\hline$j \equiv 4 \bmod 6$ & $m$ is even & $m$ is odd & $\emptyset$ & $\emptyset$ \\
\hline$j \equiv 5 \bmod 6$ & $\emptyset$ & $\emptyset$ & $k$ is even & $k$ is odd \\
\hline
\end{tabular}

\section{Fixed points and twisted invariant functionals}

Now we want to study the fixed points of the homeomorphism $\widehat{\phi}: \widehat{G} \rightarrow \widehat{G},[\rho] \mapsto$ $[\rho \phi]$ of the unitary dual. We will prove the following statement.

Lemma 4.1. There are exactly four finite-dimensional representations, which are fixed points of $\widehat{\phi}$. There is at least one infinite-dimensional representation, which is a fixed point of $\widehat{\phi}$.

Let us start from the finite-dimensional representations. As it was shown in [10] in this case there exists exactly one twisted-invariant functional on $L^{1}(G)$, or $\phi$-central $L^{\infty}$ function, coming from a twisted-invariant functional on $\rho\left(L^{1}(G)\right) \cong M(\operatorname{dim} \rho, \mathbb{C})$ (up to scaling), namely

$$
\varphi_{\rho}: g \mapsto \operatorname{Tr}(S \rho(g))
$$

where $S$ is the intertwining operator between $\rho$ and $\rho \phi$. This is an appropriate notion of character of a $\phi$-invariant representation.

First, we have to find $\mu$-invariant finite $\alpha$-orbits on $\mathbb{T}^{2}$. One can notice that

$$
\operatorname{det}\left(A^{n}-M\right)=\operatorname{det}\left(\begin{array}{cc}
a & b-1 \\
b+1 & d
\end{array}\right)=a d-b^{2}+1=\operatorname{det} A^{n}+1=2,
$$

where $A^{n}=\left(\begin{array}{ll}a & b \\ b & d\end{array}\right)$, for any $n$. Hence, the mentioned orbits are formed by points with coordinates 0 and $1 / 2$. We have 2 orbits: one of them consists of 1 point $(0,0)$ and gives rise to 1-dimensional trivial representation $\rho_{1}$, and the other consists of $A_{1}=(0,1 / 2), A_{2}=(1 / 2,0)$ and $A_{3}=(1 / 2,1 / 2)$ and gives rise to a 3 -dimensional (irreducible) representation $\rho_{2}$. Also, one has the following 1-dimensional representation $\pi$ :

$$
\pi((m, k), 2 n)=1, \quad \pi((m, k), 2 n+1)=-1 .
$$

So, we have 4 representations

$$
\rho_{1}, \quad \rho_{2}, \quad \pi, \quad \rho_{2} \otimes \pi
$$

These representations are irreducible finite dimensional (for the last one this follows from the evident observation $\left(\rho_{2} \otimes \pi\right)(g)= \pm \rho_{2}(g)$, hence the space of commuting operators is still isomorphic to $\mathbb{C}$ ). We claim that these representations give rise via (6) to 4 linear independent twisted-invariant functionals. In particular, there is no more finite-dimensional fixed points of $\widehat{\phi}$. More precisely, this follows from the general theory of [10], since these finite-dimensional representations are well separated. But we will calculate directly for the self-containing of the presentation here, and also because we need the explicit form of these functionals. 
Clearly,

$$
\varphi_{\rho_{1}} \equiv 1, \quad \varphi_{\pi}=\left\{\begin{array}{rl}
1, & \text { on } \cup_{n} L_{2 n}=B_{1} \cup B_{2}, \\
-1, & \text { on } \cup_{n} L_{2 n+1}=B_{3} \cup B_{4},
\end{array} \quad \varphi_{\rho_{2} \otimes \pi}=\varphi_{\rho_{2}} \cdot \varphi_{\pi} .\right.
$$

Let us find $\varphi_{\rho_{2}}$. In the space $L^{2}\left(A_{1}, A_{2}, A_{3}\right)$ we take the base $\varepsilon_{1}, \varepsilon_{2}, \varepsilon_{3}$ of characteristic functions in these points. One has

$$
\begin{array}{lll}
\alpha\left(\varepsilon_{1}\right)=\varepsilon_{3}, & \alpha\left(\varepsilon_{3}\right)=\varepsilon_{2}, & \alpha\left(\varepsilon_{2}\right)=\varepsilon_{1}, \\
\mu\left(\varepsilon_{1}\right)=\varepsilon_{2}, & \mu\left(\varepsilon_{2}\right)=\varepsilon_{1}, & \mu\left(\varepsilon_{3}\right)=\varepsilon_{3} .
\end{array}
$$

The representation (see $[2$, Ch. $17, \S 1]$ ) is defined by:

$$
\begin{gathered}
\rho_{2}(m, k, 0)\left(\varepsilon_{i}\right)=\chi_{A_{i}}(m, k) \cdot \varepsilon_{i}, \quad \rho_{2}(0,0, n)\left(\varepsilon_{i}\right)=\alpha^{-n}\left(\varepsilon_{i}\right)=\varepsilon_{i+n} \quad \bmod 3, \\
\rho_{2}(m, k, 0)\left(\varepsilon_{1}\right)=e^{2 \pi i(0 \cdot m+1 / 2 \cdot k)} \cdot \varepsilon_{1}=e^{\pi i k} \varepsilon_{1}, \\
\rho_{2}(m, k, 0)\left(\varepsilon_{2}\right)=e^{2 \pi i(1 / 2 \cdot m+0 \cdot k)} \cdot \varepsilon_{2}=e^{\pi i m} \varepsilon_{2}, \\
\rho_{2}(m, k, 0)\left(\varepsilon_{3}\right)=e^{2 \pi i(1 / 2 \cdot m+1 / 2 \cdot k)} \cdot \varepsilon_{3}=e^{\pi i(m+k)} \varepsilon_{3} .
\end{gathered}
$$

The representation $\widehat{\phi} \rho_{2}$ is defined by

$$
\begin{gathered}
\widehat{\phi} \rho_{2}(0,0, n)\left(\varepsilon_{i}\right)=\rho_{2}(0,0,-n)\left(\varepsilon_{i}\right)=\varepsilon_{i-n} \bmod 3, \\
\widehat{\phi} \rho_{2}(m, k, 0)\left(\varepsilon_{1}\right)=\rho_{2}(k,-m, 0)\left(\varepsilon_{1}\right)=e^{-\pi i m} \varepsilon_{1}=e^{\pi i m} \varepsilon_{1}, \\
\widehat{\phi} \rho_{2}(m, k, 0)\left(\varepsilon_{2}\right)=\rho_{2}(k,-m, 0)\left(\varepsilon_{2}\right)=e^{\pi i k} \varepsilon_{2}, \\
\widehat{\phi} \rho_{2}(m, k, 0)\left(\varepsilon_{3}\right)=\rho_{2}(k,-m, 0)\left(\varepsilon_{3}\right)=e^{\pi i(k-m)} \varepsilon_{3}=e^{\pi i(m+k)} \varepsilon_{3} .
\end{gathered}
$$

The intertwining operator is induced by $\phi$ and has the matrix $S=\left(\begin{array}{lll}0 & 1 & 0 \\ 1 & 0 & 0 \\ 0 & 0 & 1\end{array}\right)$.

Hence,

$$
\begin{gathered}
\varphi_{\rho_{2}}(m, k, n)=\operatorname{Tr}\left(S \rho_{2}(m, k, 0) \rho_{2}(0,0, n)\right)= \\
=\operatorname{Tr}\left[\left(\begin{array}{lll}
0 & 1 & 0 \\
1 & 0 & 0 \\
0 & 0 & 1
\end{array}\right)\left(\begin{array}{ccc}
e^{\pi i k} & 0 & 0 \\
0 & e^{\pi i m} & 0 \\
0 & 0 & e^{\pi i(k+m)}
\end{array}\right)\left(\begin{array}{lll}
0 & 0 & 1 \\
1 & 0 & 0 \\
0 & 1 & 0
\end{array}\right)^{n}\right]= \\
=\operatorname{Tr}\left[\left(\begin{array}{ccc}
0 & e^{\pi i k} & 0 \\
e^{\pi i k} & 0 & 0 \\
0 & 0 & e^{\pi i(k+m)}
\end{array}\right)\left(\begin{array}{lll}
0 & 0 & 1 \\
1 & 0 & 0 \\
0 & 1 & 0
\end{array}\right)^{n}\right] .
\end{gathered}
$$

For $n \equiv 0 \bmod 3$

$$
\begin{aligned}
\varphi_{\rho_{2}}(m, k, n) & =\operatorname{Tr}\left[\left(\begin{array}{ccc}
0 & e^{\pi i m} & 0 \\
e^{\pi i k} & 0 & 0 \\
0 & 0 & e^{\pi i(k+m)}
\end{array}\right)\right]=e^{\pi i(k+m)} \\
& =\left\{\begin{array}{cc}
1, & \text { if } m+k \text { is even } \\
-1, & \text { if } m+k \text { is odd }
\end{array}\right.
\end{aligned}
$$

for $n \equiv 1 \bmod 3$

$$
\varphi_{\rho_{2}}(m, k, n)=\operatorname{Tr}\left[\left(\begin{array}{ccc}
0 & e^{\pi i m} & 0 \\
e^{\pi i k} & 0 & 0 \\
0 & 0 & e^{\pi i(k+m)}
\end{array}\right)\left(\begin{array}{lll}
0 & 0 & 1 \\
1 & 0 & 0 \\
0 & 1 & 0
\end{array}\right)\right]=
$$




$$
=\operatorname{Tr}\left(\begin{array}{ccc}
e^{\pi i m} & 0 & 0 \\
0 & 0 & e^{\pi i k} \\
0 & e^{\pi i(k+m)} & 0
\end{array}\right)=e^{\pi i m}=\left\{\begin{array}{cc}
1, & \text { if } m \text { is even } \\
-1, & \text { if } m \text { is odd }
\end{array}\right.
$$

for $n \equiv 2 \bmod 3$

$$
\begin{aligned}
& \varphi_{\rho_{2}}(m, k, n)=\operatorname{Tr}\left[\left(\begin{array}{ccc}
0 & e^{\pi i m} & 0 \\
e^{\pi i k} & 0 & 0 \\
0 & 0 & e^{\pi i(k+m)}
\end{array}\right)\left(\begin{array}{lll}
0 & 1 & 0 \\
0 & 0 & 1 \\
1 & 0 & 0
\end{array}\right)\right]= \\
& =\operatorname{Tr}\left(\begin{array}{ccc}
0 & 0 & e^{\pi i m} \\
0 & e^{\pi i k} & 0 \\
e^{\pi i(k+m)} & 0 & 0
\end{array}\right)=e^{\pi i k}=\left\{\begin{array}{cc}
1, & \text { if } k \text { is even } \\
-1, & \text { if } k \text { is odd }
\end{array} .\right.
\end{aligned}
$$

$\varphi_{\rho_{2}}$ is 3-periodical in $n$, while the characteristic functions of $B_{i}$ are 6-periodical. For $j=0, \ldots, 5$ one has

$$
\begin{array}{lll}
\left.\varphi_{\rho_{2}}\right|_{B_{1} \cap L_{0}} \equiv 1,\left.\quad \varphi_{\rho_{2}}\right|_{B_{2} \cap L_{0}} \equiv-1,\left.\quad \varphi_{\rho_{2}}\right|_{B_{3} \cap L_{1}} \equiv 1,\left.\quad \varphi_{\rho_{2}}\right|_{B_{4} \cap L_{1}} \equiv-1, \\
\left.\varphi_{\rho_{2}}\right|_{B_{1} \cap L_{2}} \equiv 1,\left.\quad \varphi_{\rho_{2}}\right|_{B_{2} \cap L_{2}} \equiv-1,\left.\quad \varphi_{\rho_{2}}\right|_{B_{3} \cap L_{3}} \equiv 1,\left.\quad \varphi_{\rho_{2}}\right|_{B_{4} \cap L_{3}} \equiv-1, \\
\left.\varphi_{\rho_{2}}\right|_{B_{1} \cap L_{4}} \equiv 1,\left.\quad \varphi_{\rho_{2}}\right|_{B_{2} \cap L_{4}} \equiv-1,\left.\quad \varphi_{\rho_{2}}\right|_{B_{3} \cap L_{5}} \equiv 1, & \left.\varphi_{\rho_{2}}\right|_{B_{4} \cap L_{5}} \equiv-1,
\end{array}
$$

so $\left.\varphi_{\rho_{2}}\right|_{B_{1} \cup B_{3}} \equiv 1,\left.\varphi_{\rho_{2}}\right|_{B_{2} \cup B_{4}} \equiv-1$. the determinant of the values of the functions $\varphi_{\rho_{1}}, \varphi_{\pi}, \varphi_{\rho_{2}}, \varphi_{\rho_{2} \otimes \pi}$ on $B_{1}, B_{2}, B_{3}, B_{4}$ is

$$
\operatorname{det}\left(\begin{array}{rrrr}
1 & 1 & 1 & 1 \\
1 & 1 & -1 & -1 \\
1 & -1 & 1 & -1 \\
1 & -1 & -1 & 1
\end{array}\right)=-8 \neq 0
$$

Hence, they are linearly independent.

Nevertheless, there are infinite-dimensional irreducible $\widehat{\phi}$-invariant representations. E.g. we have a representation $\rho$ of $G$ on $L^{2}\left(\mathbb{T}^{2}\right)$ with the respect to the Lebesgue measure, with $\rho(m, k, 0)$ be the multiplier by characters in the appropriate points and $\rho(0,0,1)$ is $\alpha$, in the same manner as for $\rho_{2}$. This representation can be also obtained by inducing from the trivial representation of the subgroup $\left\{\alpha^{n}\right\} \subset G$ and Fourier transform. The irreducibility of this induced representation can also be proved using [17].

This disproves the conjecture of Fel'shtyn and Hill [6], who supposed that the Reidemeister number equals to the number of fixed points of $\widehat{\phi}$ on $\widehat{G}$

This representation is not traceable, but one can nevertheless try to calculate (6). We will do this in some fixed base, because in general operators are not of trace class. Let us choose an orthonormal base of $L^{2}\left(\mathbb{T}^{2}\right)$ formed by $\varepsilon_{s t}(x, y)=e^{2 \pi i(s x+t y)}$, $x, y \in[0,1]$. The intertwining operator $S$ is generated by $\mu$. Then

$$
\begin{gathered}
\operatorname{Tr}(S \rho(m, k, n))=\sum_{s, t} \int_{0}^{1} \int_{0}^{1}\left(\rho(m, k, 0) \rho(0,0, n) \varepsilon_{s, t}\right)(\mu(x, y)) \overline{\varepsilon_{s, t}}(x, y) d x d y= \\
=\sum_{s, t} \int_{0}^{1} \int_{0}^{1} e^{2 \pi i\langle(m, k), \mu(x, y)\rangle}\left(\rho(0,0, n) \varepsilon_{s, t}\right)(\mu(x, y)) \varepsilon_{-s,-t}(x, y) d x d y= \\
=\sum_{s, t} \int_{0}^{1} \int_{0}^{1} e^{2 \pi i\langle(m, k), \mu(x, y)\rangle} \varepsilon_{s, t}\left(\alpha^{n} \mu(x, y)\right) \varepsilon_{-s,-t}(x, y) d x d y=
\end{gathered}
$$




$$
=\sum_{s, t} \int_{0}^{1} \int_{0}^{1} e^{2 \pi i\left\langle(m, k)+\left(\alpha^{n}-\mu\right)(s, t), \mu(x, y)\right\rangle} d x d y=\sum_{(m, k)=\left(\mu-\alpha^{n}\right)(s, t)} 1 .
$$

For $n=0$ this equals 1 for $m+k$ even and 0 for $m+k$ odd. Hence, $\left.\varphi_{\rho}\right|_{B_{1} \cap L_{0}}=1$, $\left.\varphi_{\rho}\right|_{B_{2} \cap L_{0}}=0$. For $n=1$ the equality takes the form $(m, k)=(-2 s-2 t,-t)$. Hence, $\left.\varphi_{\rho}\right|_{B_{3} \cap L_{1}}=1,\left.\varphi_{\rho}\right|_{B_{4} \cap L_{1}}=0$. If $n=2 r$, the equality takes form

$$
\begin{gathered}
(m, k)=\left(\mu-\alpha^{2 r}\right)(s, t), \quad(m, k)=\left(\alpha^{r} \mu \alpha^{r}-\alpha^{2 r}\right)(s, t), \\
\alpha^{-r}(m, k)=\left(\mu-\alpha^{0}\right) \alpha^{r}(s, t) .
\end{gathered}
$$

Since $\alpha$ is an automorphism of $\mathbb{Z} \oplus \mathbb{Z}$ and by the description of $B_{i}$ via the action of $\alpha$, we obtain that $\left.\varphi_{\rho}\right|_{B_{1}}=1,\left.\varphi_{\rho}\right|_{B_{2}}=0$. Similarly, for $n$ odd. So, $\varphi_{\rho}$ is well defined and

$$
\varphi_{\rho}=\chi_{B_{1}}+\chi_{B_{3}}=\frac{1}{2}\left(\varphi_{\rho_{1}}+\varphi_{\rho_{2}}\right) \text {. }
$$

Of course, there are also $\widehat{\phi}$-invariant traceable factor representations of this group $G$, e.g. the regular representation. Since its kernel is trivial, evidently all twistedinvariant functionals can be pulled back from it.

\section{Discussion, prospectives, conjectures}

This example shows that the conjecture of [6] for general groups can survive only after eliminating badly separated points in $\widehat{G}$. A partial solution of the problem along this direction is obtained in Theorem 1.4. As it is known, the unitary dual of a group of polynomial growth is well separated, this gives hope to get an analytical proof of a version of twisted Burnside theorem for these groups. Also, while the present paper was under refereeing A.F. and E.T. have obtained the following general theorem: for any automorphism of any almost polycyclic group $R(\phi)=S_{f}(\phi)$ if one of them is finite, where $S_{f}(\phi)$ is the number of fixed points of $\widehat{\phi}$ on the finite-dimensional part of $\widehat{G}$ ([9], see also the survey [8] for a number of related statements, counterexamples, etc.).

\section{References}

[1] J. Arthur and L. Clozel, Simple algebras, base change, and the advanced theory of the trace formula. Princeton University Press, Princeton, NJ, 1989. MR 90m:22041

[2] A. O. Barut and R. Rączka, heory of group representations and applications, second ed. World Scientific Publishing Co., Singapore, 1986. MR 88c:22013

[3] J. Dauns and K. H. Hofmann, Representations of rings by continuous sections. Mem. Amer. Math. Soc., vol. 83, Amer. Math. Soc., Providence, RI, 1968.

[4] A. Fel'shtyn, Dynamical zeta functions, Nielsen theory and Reidemeister torsion, Mem. Amer. Math. Soc. 147 (2000), no. 699, xii+146. MR 2001a:37031

[5] A. Fel'shtyn and D. Gonçalves, Reidemeister numbers of Baumslag-Solitar groups, E-print arXiv:math.GR/0405590, 2004, (to appear in Algebra Discrete Math.).

[6] A. Fel'shtyn and R. Hill, The Reidemeister zeta function with applications to Nielsen theory and a connection with Reidemeister torsion, K-Theory 8 (1994), no. 4, 367-393. MR 95h:57025

[7] _ Dynamical zeta functions, congruences in Nielsen theory and Reidemeister torsion, Nielsen theory and Reidemeister torsion (Warsaw, 1996), Polish Acad. Sci., Warsaw, 1999, pp. 77-116. MR 2001h:37047

[8] A. Fel'shtyn and E. Troitsky, Geometry of Reidemeister classes and twisted Burnside theorem, (to appear in K-Theory).

[9] _ , Twisted Burnside theorem, Preprint 46, Max-Planck-Institut für Mathematik, 2005. 
[10] _ A twisted Burnside theorem for countable groups and Reidemeister numbers, Proc. Workshop Noncommutative Geometry and Number Theory (Bonn, 2003) (K. Consani, M. Marcolli, and Yu. Manin, eds.), Vieweg, Braunschweig, 2006, pp. 141-154, (Preprint MPIM2004-65).

[11] A. L. Fel'shtyn, The Reidemeister number of any automorphism of a Gromov hyperbolic group is infinite, Zap. Nauchn. Sem. S.-Peterburg. Otdel. Mat. Inst. Steklov. (POMI) 279 (2001), no. 6 (Geom. i Topol.), 229-240, 250. MR 2002e:20081

[12] D. Gonçalves and P. Wong, Twisted conjugacy classes in exponential growth groups, Bull. London Math. Soc. 35 (2003), no. 2, 261-268. MR 2003j:20054

[13] A. Grothendieck, Formules de Nielsen-Wecken et de Lefschetz en géométrie algébrique, Séminaire de Géométrie Algébrique du Bois-Marie 1965-66. SGA 5, Lecture Notes in Math., vol. 569, Springer-Verlag, Berlin, 1977, pp. 407-441.

[14] B. Jiang, Lectures on Nielsen fixed point theory, Contemp. Math., vol. 14, Amer. Math. Soc., Providence, RI, 1983.

[15] A. A. Kirillov, Elements of the theory of representations. Springer-Verlag, Berlin Heidelberg New York, 1976.

[16] G. Levitt and M. Lustig, Most automorphisms of a hyperbolic group have very simple dynamics., Ann. Scient. Éc. Norm. Sup. 33 (2000) 507-517.

[17] M. Saito, Représentations unitaires monomiales d'un groupe discret, en particulier du groupe modulaire, J. Math. Soc. Japan 26 (1974), no. 3, 464-482.

[18] S. Shokranian, The Selberg-Arthur trace formula, Springer-Verlag, Berlin, 1992, Based on lectures by James Arthur. MR 93j:11029

[19] E. Troitsky, Noncommutative Riesz-Markov-Kakutani theorem and weak twisted Burnside theorem, Funct. Anal. Appl. 40 (2006), no. 2, 44-54.

Instytut Matematyki, Uniwersytet Szczecinski, Ul. Wielkopolska 15, 70-451 Szczecin, Poland and Department of Mathematics, Boise State University, 1910 University Drive, BoISE, IDAHO, 83725-155

E-mail address: felshtyn@diamond.boisestate.edu, felshtyn@mpim-bonn.mpg.de

URL: http://www.mat.univ.szczecin.pl/en/staff/felshtyn_a.html

Dept. of Mech. and Math., Moscow State University, 119992 GSP-2 Moscow, Russia

E-mail address: troitsky@mech.math.msu.su

$U R L:$ http://mech.math.msu.su/ ${ }^{\sim}$ troitsky

St.Petersburg Department of Steklov Institute of Mathematics, 27 Fontanka, St. PeTERSBURG 191011, RUSSIA

E-mail address: vershik@pdmi.ras.ru

$U R L$ : http://www.pdmi.ras.ru/〜vershik 